\title{
THE INFLUENCE OF COMORBID PATHOLOGY ON THE CARDIOVASCULAR CONDITION IN YOUNG SPORTSMEN
}

Nikolay Cherkasov

\author{
Astrakhan State Medical University, Astrakhan, Russia \\ kafedra1@mail.ru
}

\begin{abstract}
A B STRACT -74 young athletes aged 8-10 years underwent cardiovascular assessment. Additionally, levels of $\mathrm{MB}-$ creatine phosphokinase, troponin - $\mathrm{T}$, parameters of heart rate variability were studied. It is stated that the syndrome of vegetative dysfunction and undifferentiated connective tissue dysplasia are the adverse background contributing to the development of disturbances of cardiac activity, arrhythmias and cardiomyopathies in young athletes.
\end{abstract}

KEY WORDS - children involved in sports, biochemistry indicators: creatine phosphokinase, troponin-T, spectral indicators of heart rate variability, syndrome of vegetative dysfunction, undifferentiated connective tissue displasia, comorbid pathology

In recent years the concept of comorbidity has been included in clinical pediatric practice. There are scientific papers on comorbid pathology in children with bronchopulmonary dysplasia, congenital pathology of the musculoskeletal system, undifferentiated connective tissue dysplasia, nephropathy and other $[1,2,4,5,6]$. The evaluation of disorders of the cardiovascular system without comorbidity among young sportsmen should not be objective.

In children (adolescents) involved in sports, undoubtedly, various violations of the vegetative regulation affecting the condition of health can occur with great frequency. There is not seldom the combination of manifestations of undifferentiated connective tissue dysplasia with autonomic dysfunction. The comorbidity of these symptoms and cardiac abnormalities among young sportsmen remains to be not enough investigated.

It is generally considered that to estimate the degree of myocardium damage (cardiomyocytes), the determination of the activity of the MV fraction of creatinine phosphokinase (MB-CPK) of the troponinT level (Tr-T), etc. may be used $[3,4,7,8]$.

The study of the state of the spectral parameters of heart rate variability (HRV) is not only a significant addition to the electrocardiographic data but also allows to quantify the vegetative influence on the work of various regulatory systems.

The utilized activities of MB-CPK and the main parameters of heart rate variability allow to identify and evaluate various signs of impaired cardiac activity in young sportsmen. The search of clinical, biochemical and instrumental criteria for myocardial dysfunction and heart rhythm disturbances in comorbid pathology in young sportsmen is an important unsolved problem in pediatrics.

The aim of investigation: is to establish the value of comorbid pathology in the development of various forms of cardiac abnormalities in young sportsmen.

\section{THE CHARACTERISTIC OF CHILDREN}

Under the supervision there were 74 children, aged $8-10$ years (average age $9 \pm 1$ ), engaged over 3 years and more in handball. The sportsmen involved in other sports with organic pathology of the cardiovascular system and having been undergone the acute respiratory diseases $1-1.5$ months before the examination were excluded.

According to results of the clinical examination the respondents were divided into three groups. The first group included 28 children with signs of undifferentiated connective tissue dysplasia (UCTD).

The second group included 19 children with the manifestation of the syndrome of vegetative dysfunction (SVD) of the mixed character in the compensation stage. 13 children had vagotonia (asimpathicotonia).

In the third group, there were 27 young sportsmen with signs of UCTD combined with ADD of mixed character in the period of acute attack of the disease.

\section{THE METHODS OF INVESTIGATION}

The investigation was conducted on the base of the Regional Curative Physical -Culture Dispensary of Astrakhan. It included the collection of data for medical history about the peculiarities of child development and diseases of the circulatory system. The pediatrician and pediatric cardiologist monitored all 
children for 2-3 times a year. Alongside with the clinical evaluation of the cardiovascular system, ELISA and troponin- $T$ content there were studied all the observed levels of MB-CPK activity using the BeringerMaugeim enzyme-test system.

From instrumental methods, standard electrocardiography (ECG), echocardiography (ECHO-CG) were used with the interpretation of the data obtained according to standards. To study the vegetative and energy characteristics of the organism, there were used the definition of the main spectral parameters of heart rate variability (HRV) on the Polyspectra-12E apparatus of the Neyrosoft Company. The obtained data were processed according to generally accepted methods of variation statistics.

\section{RESULTS AND DISCUSSION}

USTD in the first group was characterized by the presence of asthenic physique (11), hypermobility of joints (8), chest deformity (5), scoliosis (6), flatfoot (5). From the visceral signs on the echocardiogram there were determined: mitral valve prolapse with I-II degree regurgitation, an additional chord of the left ventricle; prolapse of the tricuspid valve with regurgitation of grades I-II, 5 had diastolic disfunction, 7 had the tendency to decrease in systolic function. These visceral manifestations can be interpreted as dysplastic heart. The boundaries of relative cardiac dullness did not change significantly. During auscultation, in 9 observables there were determined muffled tones of the heart (4), attenuation of I tone at point V (2). On the ECG 13 persons showed signs of impaired repolarization.

The activity of MB-CPK $(p<0,05)$ and levels, $\operatorname{Tr}-\mathrm{T}(\mathrm{p}<0,05)$ were moderately elevated in contrast to the norm. These clinical-biochemical and instrumental data given above allowed to make the diagnosis of secondary cardiomyopathy. It was caused by the comorbid state of USTD and the influence of physical activity in sports (Table 1 ).

In the second group young sportsmen had no complaints. Clinical signs of SVD were detected during careful examination: red dermographism, skin moisture, lethargy, fatigue and bradycardia at rest. The boundaries of relative cardiac dullness in 9 sportsmen were moderately widen to the left. Heart sounds were moderately muffled in 10 , soft systolic murmur at point $\mathrm{V}(5)$. In 17 children sinus bradyarrhythmia was determined, in 7 children there were ventricular premature beats. CM ECG confirmed these data. In this case bradyarrhythmia occurred in one third part at night from 40 to 54 beats per minute.

The levels of biochemical indicators of myocardial damage did not significantly differ from the norm $(\mathrm{p}>0,05, \mathrm{p}>0,05)$ (Table 1$)$.
In comparison of findings of clinical and instrumental examination, the diagnosis of cardiac rhythm disturbance, sinus bradyarrhythmia (8\%), over ventricular $(10 \%)$ or ventricular premature beats $(5 \%)$ on the background of SVD was made. The occurrence of these disorders should be regarded as comorbide pathology of SVD and heart rhythm disturbance in physical exertion and emotional stress.

In the $3^{\text {rd }}$ group (27) children had complaints of recurrent heart pain. Phenotypic signs of UCTD were noted - hypermobility of joints, flat-footedness, violation of posture, asthenic physique, deformity of auricles, nails, etc. Each observed person had up to six or more signs in combination with 2-3 visceral ones from the side of the heart (red dermographism, lethargy, fatigue, bradycardia at rest, skin moisture) of the SVD had mixed the character with marked manifestations.

The boundaries of relative cardiac dullness were moderately widen to the left in 7 children. Muffled heart sounds at 8 , soft systolic murmur at the apex and at point $\mathrm{V}(7)$. The sinus bradyarrhythmia in 8 , supraventricular premature beats in 5, ventricular in 3 . The levels MB-CPK, troponin-T were high in comparison with data the firat and second groups (Table 1).

On ECG disorders of repolarization in 15, rhythm disturbances confirmed the clinical data. At the CM-ECG supraventricular extrasystoly was recorded in 5 , ventricular in 3 and in 8 cases-sinus bradyarrhythmia.

On the ECHO-CS the contractility of the myocardium was not changed; in 7, the decrease of left ventricular diastolic function was determined.

These data indicate the presence of combinations of comorbide diseases in this group such as: UCTD and SVD. The influence of prolonged physical exertion in combination with emotional stress they have become the cause of the development of cardiac pathology: secondary cardiomyopathy and cardiac arrhythmia.

Thus the presence of undifferentiated connective tissue dysplasia, autonomic disfunction syndrome in sportsmen is an unfavourable background for the development of cardiovascular pathology during athletic exercises in children. Prognosticallyunfavourable sign of the development of cardiac abnormalities is autonomic disfunction syndrome. The asympathicotonia may contribute to the formation of cardiac arrhythmia or the manifestation of existing latent forms.

The combinations of UCTD with autonomic disfunction syndrome in young sportsmen often lead to the development of cardiac abnormalities.In these cases the combinations of myocardial disfunction with cardiac rhythm disturbances of functional and organic 
Table 1. The value of laboratory and instrumental data in young sportsmen

\begin{tabular}{|c|c|c|c|}
\hline \multirow[t]{2}{*}{ Indicators } & \multicolumn{3}{|l|}{ Groups } \\
\hline & First $(n=28)$ & Second $(n=19)$ & Third $(n=27)$ \\
\hline MB-CPK (F/I) & $54,3 \pm 0,4^{* * \# \#}$ & $34,5 \pm 0,3^{*} \# \#$ & $58,5 \pm 0,4^{* *}$ \\
\hline Troponin-T (mg/l) & $0,044 \pm 0,006^{*} \# \#$ & $0,045 \pm 0,005^{*} \# \#$ & $0,052 \pm 0,006^{* *}$ \\
\hline $\mathrm{TP}\left(\mathrm{me}^{2}\right)$ & $2510 \pm 544^{*} \#$ & $3150 \pm 554^{* * \# \#}$ & $2120 \pm 560^{*}$ \\
\hline $\operatorname{VLF}\left(\mathrm{Me}^{2}\right)$ & $1234 \pm 418^{*} \#$ & $2910 \pm 560^{* * \#}$ & $1240 \pm 420^{*}$ \\
\hline $\operatorname{LF}\left(\mathrm{Me}^{2}\right)$ & $920 \pm 134^{* *} \# \#$ & $950 \pm 610^{* *} \#$ & $915 \pm 136^{*}$ \\
\hline $\mathrm{HF}\left(\mathrm{Me}^{2}\right)$ & $640 \pm 152^{*} \#$ & $690 \pm 148^{*} \# \#$ & $651 \pm 144^{*}$ \\
\hline
\end{tabular}

Legend: $p^{*}$ in the comparison of all groups with the norm $p^{*}>0,05 p^{* *}<0,05$ $p$ - in comparing the first and second groups with the third $p>0,05 p<0,05$

nature are possible. The physical and emotional stress in sportsmen should lead to the development of functional disorders of the heart or the manifestation of latent disfunction. The presence of comorbid pathology: UCTD and SVD may be the risk factors for the development of cardiac abnormalities in the formation of arrhythmia and cardiomyopathy in young sportsmen.

\section{REFERENCES:}

1. Belyalova F.I. The treatment of internal diseases in the condition of comorbidity // F.I. Belyalova Irkutsk: Irkutsk State Institute for doctors' perfection. 2011.305p

2. Komissarova O.A. Criteria of heart damage activity in children with congenital heart disease // O.A. Komissarova, T.N. Doronina, N.S. Cherkasov, M.Ya. Ledyaev, L.K. Yavikova // Vestnik of Volgograd. 2012, №2, P. 39-41.

3. Doronina T.N. The Clinico-biochemical criteria of cardio adaptation of children // T.N. Doronina, N.S. Cherkasov // Archiv Euro Medica. Vol. 3. №1, 2013. P. 61-63.
4. Doronina N.N., Cherkasov N.S. Zhidovinov A.A. The Peculiarities of myocardial condition estimation in interatrial septal defects in children of early age // Achiv Euro Medica. 2016. Vol. 6, №2, P. 27-29.

5. Cherkasov N.S. The heart of a child // Astrakhan. Publishing House of the Astrakhan State Medical University. 2015. 184 p.

6. Cherkasov N.S.,Doronina T.N. Peculiar Features of heart activity metabolic correction in children and adolescent athletes // Archiv Euro Medica. 2018 Vol. 8. №1. P. 69-71.

7. Cherkasov N.S., Doronina T.N., Prakhov A.V. The significance of determination body's energy supply before and after competition among young athletes // Archiv EuroMedica. 2019. Vol. 9. №1. P. 146-148. $10.35630 / 2199-885 X / 2019 / 9 / 1 / 146$

8. VertKin A.A. Comorbidity - a new pathology. Technology of its prophylaxis and treatment // A.A. Vertkin, N.O. Khovasova //Arhivinternal medicine. 2013. № 4, P.68. 